\title{
A Qualidade da Experiência de Estágio e o Desenvolvimento Vocacional de Estudantes dos Cursos Tecnológicos
}

\author{
Work Experience Quality and Vocational Development of Students \\ from Technological Courses
}

\author{
Vitor Manuel Gamboa ${ }^{*}, a$ \& Maria Paula Paixão ${ }^{b}$ \\ ${ }^{a}$ Universidade do Algarve, Faro, Distrito de Faro, Portugal \\ $\&{ }^{b}$ Universidade de Coimbra, Coimbra, Distrito de Coimbra, Portugal
}

\begin{abstract}
Resumo
A aprendizagem em contexto real de trabalho constitui uma importante componente da formação dos alunos dos cursos tecnológicos. No que se refere ao efeito deste tipo de experiências no desenvolvimento vocacional dos alunos, os resultados das investigações não são muito conclusivos. Por esta razão, recorrendo a um desenho longitudinal (pré e pós-estágio), este estudo teve como principal objetivo analisar a relação entre a qualidade do estágio e o desenvolvimento vocacional dos alunos do ensino tecnológico $(N=196)$. Os resultados obtidos sugerem a relevância da qualidade da experiência de trabalho no desenvolvimento vocacional dos alunos, sobretudo no âmbito do processo de exploração. Palavras-chave: Desenvolvimento vocacional, exploração de carreira, tomada de decisão, compromisso de carreira, qualidade do estágio.
\end{abstract}

\begin{abstract}
The provision of workplace-based experiences (internship) is an important component of training programs of students attending vocational education courses. Regarding the impact of such experiences in the vocational development, results of the present research are not conclusive enough. Therefore, the main purpose of this study is to clarify the relationship between work experience quality and students vocational development. Using a longitudinal design, we conducted a study that explores the relationship between the perceived qualities of the training experience and the vocational development of Portuguese high school students $(N=196)$. The results suggest that the work experience quality is relevant for the vocational development of students, mainly, in what concerns the process of career exploration.

Keywords: Vocational development, career exploration, career decision-making, career commitment, internship quality.
\end{abstract}

O estágio curricular constitui uma componente importante do plano de formação dos jovens que frequentam os cursos tecnológicos do ensino secundário português $\left(10^{\circ}, 11^{\circ}\right.$ e $12^{\circ}$ anos de escolaridade). Enquanto medida de política educativa, a generalização desta modalidade de aprendizagem experiencial a todos os percursos profissionalizantes parece sugerir que, por si só, a aprendizagem desenvolvida em contexto meramente académico não garante a preparação dos estudantes, necessitando esta de ser complementada com as aprendizagens que só o contexto real de trabalho pode proporcionar. É neste sentido que se observa, quer nos países desenvolvidos, quer nos países em vias de desenvolvimento, um esforço crescente para tornar os currículos mais adequados às mudanças que vão ocorrendo no mercado de trabalho (Halpern, 2012; Larson,

*Endereço para correspondência: Faculdade de Ciências Sociais e Humanas, Universidade do Algarve, Campus de Gambelas, Faro, Portugal 8005-139.E-mail:vgamboa@ ualg.pt e mppaixao@fpce.uc.pt
Wilson, \& Mortimer, 2002), constituindo a organização de experiências de trabalho a principal estratégia de conexão entre a escola e a realidade ocupacional (Griffiths $\&$ Guile, 2004). No que se refere ao efeito deste tipo de experiências no desenvolvimento vocacional, os resultados da investigação não são muito consistentes, ainda que o corpo teórico da Psicologia Vocacional reforce claramente a expectativa de que a aprendizagem em contexto real de trabalho pode ter um impacto significativo no desenvolvimento vocacional dos jovens (e.g., Dawis, 2005; Lent, Brown, \& Hackett, 1994; Savickas, 2005; Super, Savickas, \& Super, 1996; Vondracek, Lerner, \& Schulenberg, 1986), sobretudo quando se trata do primeiro contacto formal e estruturado com uma realidade que tem vindo a perder visibilidade junto dos mesmos (Csikszentmihaly \& Schneider, 2000). No campo dos estudos empíricos, por seu turno, foram reunidas diversas evidências de que a qualidade das experiências de trabalho tem uma influência considerável no desenvolvimento global e vocacional dos estudantes (Brooks, Cornelius, Greefield, \& Joseph, 1995; Blustein, 
1997a; Carless \& Prodan, 2003; Johnson \& Mortimer, 2002; Kuijpers \& Meijers, 2012; Mortimer, 2003; Rogers \& Creed, 2011; Stone \& Mortimer, 1998; Watts, 1996), embora não se tenha clarificado se esse impacto se faz de forma uniforme ou se existirão facetas cujo efeito é mais pronunciado. Por esta razão, autores como Creed e Patton (2003) e Skorikov e Vondracek (1997) sugerem que a investigação futura deve procurar relacionar indicadores do desenvolvimento vocacional com a natureza (qualidades) das experiências de trabalho.

\section{Qualidade da Experiência de Estágio e o Processo de Exploração Vocacional}

A exploração vocacional, enquanto processo psicológico complexo de exploração do mundo e de si próprio (Jordaan, 1963; Porfeli \& Lee, 2012; Taveira, 2001), é um garante da adaptabilidade na carreira (Blustein, 1997b; Savickas, 2005) que parece ter particular importância naqueles períodos de transição em que os indivíduos se vêem confrontados com o desempenho de novos papéis (Blustein, 1997b; Flum \& Blustein, 2000; Jordaan, 1963; Kalakoski \& Nurmi, 1998; Taveira, 2001). No que se refere à realização de uma experiência de aprendizagem em contexto real de trabalho, esta atividade tipifica uma situação que, segundo autores como Blustein e Flum (1999), Jordaan (1963) e Kalakoski e Nurmi (1998), pode dar lugar a um significativo incremento da atividade exploratória, uma vez que a adaptação e o desempenho dos alunos parecem depender da informação que os mesmos são capazes de recolher relativamente ao novo contexto de aprendizagem, designadamente no que diz respeito às novas regras de trabalho, às expectativas dos professores $\mathrm{e}$ supervisores, e às exigências técnicas e relacionais contidas no papel de estagiário. Neste âmbito, o alargamento da atividade exploratória ao novo contexto de aprendizagem, permite que os alunos recém-chegados enriqueçam o seu repertório comportamental, de forma a serem capazes de responder satisfatoriamente às solicitações e aos desafios que o mesmo apresenta.

Admitindo a relação anteriormente explicitada, Blustein (1997a) e Jordaan (1963) consideram que a exploração vocacional, na qualidade de processo que atualiza a relação entre o self e o mundo, pode efetivamente constituir um meio privilegiado na construção dos novos significados que decorrem da experiência de aprendizagem em contexto real de trabalho, ainda que essa construção não dependa apenas das atitudes e competências daquele que explora, mas, também, das condições oferecidas pelo contexto, nomeadamente, no que se refere à diversidade de tarefas, à autonomia experimentada, à supervisão e às relações sociais e de amizade desenvolvidas (e.g., Blustein, 1997a; Blustein \& Flum, 1999; Blustein, Prezioso, \& Schultheiss, 1995; Ryan \& Deci, 2000), donde se depreende que nem todas as situações de formação em contexto real de trabalho serão igualmente facilitadoras das aprendizagens e da exploração vocacional. A literatura sugere, de forma consistente, que o suporte proporcionado no contexto de trabalho permite reduzir a ansiedade e o stresse experimentado neste tipo de situações, estimula a procura de apoio, aumenta a confiança na realização de novas tarefas e facilita a partilha de experiências (e.g., Blustein et al., 1995; Hirschi, 2009; Hirschi, Niles, \& Akos, 2011; Kenny \& Bledsoe, 2005), sendo que neste contexto deve ser destacado o papel do supervisor e dos professores orientadores (e.g., Cheung \& Arnold, 2010; Creed, Fallon, \& Hood, 2009; Kenny \& Bledsoe, 2005). Juntamento com o suporte, os contextos devem ser desafiantes, promover a autonomia, apresentar um grau de estruturação adequado e permitir a aquisição de novas competências (Ryan \& Deci, 2000). Para além das qualidades já referidas, a exploração vocacional parece beneficiar também com os processos de balanço e de reflexão relativos à experiência de aprendizagem realizada em contexto real de trabalho, o designado "debriefing" (e.g., Kuijpers, Meijers, \& Gundy, 2011; Petherbridge, 1996; Watts, 1996).

\section{Qualidade da Experiência de Estágio e os Processos de Tomada de Decisão e Compromisso de Carreira}

Tal como acontece com o processo de exploração vocacional, também quando procuramos abordar a influência das experiências de trabalho na tomada de decisão e no compromisso de carreira, estamos, num primeiro momento, a admitir a relevância das dimensões relacional e contextual destes processos. A este propósito, Bright, Pryor, Wilkenfeld e Earl (2005) advertem que as decisões não se tomam no vazio e que, por essa razão, não considerar as dimensões contextuais na tomada de decisão e no compromisso de carreira é limitar muito a nossa compreensão relativamente a estes processos. Contudo, a inserção no contexto real de trabalho não terá uma tradução direta e imediata no processo decisório dos alunos, sendo necessário que estes sejam capazes de retirar desta experiência de aprendizagem as evidências necessárias à progressão na tomada de decisão, relacionando-as com os seus interesses, valores e competências, no âmbito de um exercício de reflexão mais introspetivo, indispensável à clarificação e reestruturação do self (e.g., Porfeli \& Lee, 2012; Savickas, 2005). A adoção desta linha conceptual remete-nos, mais uma vez, para a importância da qualidade do contexto de trabalho (ou de aprendizagem) no desenvolvimento das competências de tomada de decisão e de compromisso na carreira, tão necessárias numa fase que corresponde ao início da atividade profissional de uma parte significativa dos alunos (e.g., Vianen, Pater, \& Preenen, 2009). No domínio da investigação empírica, os estudos que procuraram analisar a relação entre experiências de trabalho e a decisão e compromisso de carreira foram de facto muito pouco conclusivos (e.g., Skorikov \& Vondracek, 1997). Em alguns casos, a experiência de trabalho parece aumentar o nível de certeza com a decisão já tomada (e.g., Carless \& Prodan, 2003; Rogers \& Creed, 2011), enquanto noutros o efeito parece ser o inverso (e.g., Borges, 2007), sendo que 
a principal questão reside na necessidade de se identificar quais as dimensões contextuais (qualidades) que melhor explicam os efeitos eventualmente observados. De uma forma geral, tal como na secção relativa à exploração, são os contextos de trabalho mais ricos em oportunidades de aprendizagem (e.g., Aryee \& Tan, 1992) e com uma forte suporte relacional, sobretudo da parte do supervisor (e.g., Blau, 1985), que maior impacto têm na progressão no processo de tomada de decisão e compromisso de carreira. Neste âmbito, esclarecer a relação entre as diferentes qualidades do estágio curricular e o desenvolvimento vocacional dos alunos dos cursos Tecnológicos passa a constituir o principal objetivo do presente estudo.

\section{Método}

\section{Participantes}

Participaram no estudo 196 estudantes portugueses, de ambos os sexos (129 rapazes, 65,8\%; 67 raparigas, 34,2 $\%$ ), matriculados no $12^{\circ}$ ano de escolaridade e que realizaram o estágio curricular (ET) dos respectivos cursos no ano letivo 2007/2008. No que se refere à idade, os valores oscilam entre os 16 e os 22 anos, situando-se a média nos 17.81 anos $(D P=1,02)$. O estágio dos alunos que participaram neste estudo teve uma duração de 226 horas. Quanto à trajetória escolar anterior, $41,3 \%$ dos alunos refere já ter reprovado pelo menos uma vez, no decurso da sua trajetória escolar. Quanto ao nível socioeconómico, a maior parte dos participantes, cerca de $52 \%$, situa-se no nível médio.

\section{Instrumentos}

Questionário de dados sócio-demográficos - serviu para caracterizar os participantes no que se refere aos seguintes aspetos: curso, sexo, idade, habilitações literárias e profissão dos progenitores, profissão do pai e da mãe e trajetória escolar anterior.

Career Exploration Survey (CES; Stumpf, Colarelli, \& Hartman, 1983; Taveira, 1997). Trata-se de uma versão portuguesa da Career Exploration Survey, que tem como principal finalidade proporcionar uma medida multidimensional do processo de exploração vocacional nos seus três componentes principais: as crenças de exploração, os comportamentos de exploração e as reações de exploração vocacional (Taveira, 1997, 2001). A versão portuguesa é composta por 54 itens, válidos para avaliar doze das dezesseis dimensões originais da escala. Nos itens 1 a 43, as respostas são dadas numa escala do tipo Likert com 5 pontos, na qual o 1 significa muito poucas vezes, ou muita pouca, e o 5 corresponde a muitas vezes, ou muitíssima. Por sua vez, nos itens 44 a 51, que dizem respeito às dimensões stresse com a exploração e stresse com a tomada de decisão, as respostas passam a ser dadas numa escala de tipo Likert com 7 pontos, na qual 1 corresponde ao experienciar de uma tensão mínima, enquanto o 7, pelo contrário, traduz muita tensão perante as situações apresentadas. A validade, fidelidade e multidimensionalidade da
CES já foram amplamente demonstradas, tanto na versão original (Stumpf et al., 1983), como na versão portuguesa (e.g., Taveira, 1997).

Career Decision Scale (CDS; Osipow, Carney, Winer, Yanico, \& Koschier, 1976; Silva, 1997). Organizada em duas escalas (Certainty Scale e Indecision Scale), permite identificar as razões que possam estar na base das dificuldades na tomada de decisão da carreira. As respostas aos itens, de ambas as subescalas, são dadas numa escala de tipo Likert, com quatro posições. A soma dos itens 3 a 18 permite obter um índice global de indecisão, que oscila entre 16 e 64 , sendo que os valores mais elevados refletem um maior grau de indecisão vocacional. No que se refere às características psicométricas da $\mathrm{CDS}$, vários estudos atestaram a sua fidelidade e validade, ao longo das últimas três décadas (e.g., Osipow et al., 1976; Osipow \& Winer, 1996; Silva, 1997; Taveira, 1997).

Commitment to Career Choices Scale (CCCS; Blustein, Ellis, \& Devenis, 1989; Silva, 1997) - trata-se de uma escala que operacionaliza o modelo bidimensional do processo de compromisso com as escolhas de carreira, desenvolvido e confirmado por Blustein et al. (1989): (a) Compromisso com as Escolhas de Carreira (VECS; 19 itens), que reflete o nível de compromisso dos indivíduos com as suas decisões da carreira; e (b) Tendência para a Exclusão de Opções (TTFS; 8 itens), que identifica as diferenças individuais no modo como os indivíduos se comprometem com as suas decisões de carreira. Os participantes assinalam o seu nível de concordância com os itens, usando uma escala de 7 pontos (1-Nunca é verdadeiro para mim, a 7-Sempre verdadeiro para mim). As pontuações mais baixas na VECS traduzem um maior grau de compromisso com as escolhas de carreira, enquanto os resultados mais elevados na TTFS revelam uma forte predisposição para realizar compromissos prematuramente. No que se refere às características psicométricas, ambas as escalas provaram ser bastante estáveis (teste-reteste); quer num intervalo de duas semanas (TTFS - 0,82; VECS - 0,90), quer num intervalo de quatro semanas (TTFS $-0,84$; VECS - 0,92); e precisas (VECS - 0,92, TTFS - 0,82; Blustein et al., 1989).

Inventário da Qualidade do Estágio. Trata-se de um instrumento de autorresposta construído pelos autores deste estudo, que tem como principal objetivo avaliar as perceções dos alunos dos cursos Tecnológicos do ensino secundário, em nove dimensões relativas à qualidade dos estágios curriculares: (a) Autonomia $(\alpha=0,81)$, (b) Feedback dos Colegas $(\alpha=0,83)$, (c) Suporte Social $(\alpha=0,82)$, (d) Variedade de tarefas $(\alpha=0,83)$, (e) Oportunidades de aprendizagem $(\alpha=0,80)$, (f) Clareza das instruções do supervisor $(\alpha=0,79)$, (g) Treino proporcionado pelo supervisor $(\alpha=0,84)$, (h) Feedback do supervisor $(\alpha=$ 0,80 ), (i) Suporte e encorajamento do supervisor ( $\alpha=$ $0,87)$. As respostas são dadas numa escala do tipo Likert com cinco pontos, na qual o 1 significa discordo bastante e o 5 corresponde a concordo bastante. 


\section{Desenho do Estudo e Procedimentos de Recolha e Análise dos Dados}

No presente estudo optámos por um desenho longitudinal de curta duração, com dois momentos de recolhas de dados intervalados por um período de treze semanas. Depois de obtidas as devidas autorizações e o consentimento dos participantes, os instrumentos foram aplicados, em contexto de sala de aula, pelo primeiro autor do estudo (antes e depois do estágio). Para garantir a confidencialidade dos dados, o emparelhamento dos questionários fez-se com recurso a um código alfanumérico. Quanto aos procedimentos de análise dos dados, depois da estatística descritiva e do estudo das correlações, recorremos a análises de variância e ao test $t$ student, a fim de se testar a existência de diferenças nas variáveis vocacionais em função das características sócio-demográficas dos participantes e dos momentos de recolha de dados. A etapa seguinte na análise dos dados procurou responder à principal finalidade deste estudo - conhecer o efeito da qualidade do estágio nas mudanças ocorridas na exploração vocacional, na tomada de decisão e no compromisso de carreira. Para tal, calcularam-se equações de regressão (hierárquica), nas quais as medidas vocacionais em T2 (2. ${ }^{\circ}$ momento) entraram na qualidade de variáveis dependentes, enquanto as mesmas medidas em T1 (1. ${ }^{\circ}$ momento), foram incluídas num primeiro bloco, de modo a se controlar o seu efeito. Por último, importa referir que o nível utilizado para avaliar a significância dos testes estatísticos efetuados foi de 0,05 . As análises foram realizadas com recurso ao programa de tratamento de dados estatísticos SPSS (Statistical Package for the Social Sciences), versão 17.

Tabela 1

Médias e Desvios-padrão das Variáveis em Estudo para Ambos os Momentos, Testes $t$ de Student para Amostras Emparelhadas e Magnitude do Efeito $(N=196)$.

\begin{tabular}{|c|c|c|c|c|c|c|c|}
\hline & \multicolumn{2}{|c|}{$1^{\circ}$ Momento } & \multicolumn{2}{|c|}{$2^{\circ}$ Momento } & \multirow[b]{2}{*}{$t$} & \multirow[b]{2}{*}{$p$} & \multirow[b]{2}{*}{$d$} \\
\hline & Média & $D P$ & Média & $D P$ & & & \\
\hline Indecisão (CDS) & 34,45 & 8,019 & 36,21 & 8,419 & $-3,012$ & 0,003 & 0,21 \\
\hline \multicolumn{8}{|l|}{ CCCS: } \\
\hline Tendência exclusão opções (TTF) & 36,98 & 7,423 & 37,48 & 7,150 & $-0,842$ & 0,401 & 0,07 \\
\hline Exploração e compromisso (VEC) & 70,38 & 16,467 & 71,95 & 14,533 & $-1,523$ & 0,129 & 0,11 \\
\hline \multicolumn{8}{|l|}{ CES: } \\
\hline Estatuto do Emprego (EE) & 9,08 & 2,193 & 9,62 & 2,068 & $-3,328$ & 0,001 & 0,25 \\
\hline Certeza resultados Exploração (CR) & 7,49 & 2,797 & 8,40 & 2,673 & $-4,631$ & 0,000 & 0,33 \\
\hline Instrumentalidade Externa (IE) & 33,43 & 5,202 & 33,38 & 5,062 & 0,140 & 0,889 & 0,01 \\
\hline Instrumentalidade Interna (II) & 14,29 & 2,586 & 14,22 & 2,251 & 0,341 & 0,734 & 0,03 \\
\hline Importância de obter Posição Preferida (IPP) & 11,34 & 2,251 & 10,92 & 2,219 & 2,487 & 0,014 & 0,19 \\
\hline Exploração do Meio (EM) & 11,17 & 3,231 & 12,33 & 3,197 & $-4,637$ & 0,000 & 0,33 \\
\hline Exploração de Si próprio (ES) & 14,60 & 3,695 & 15,59 & 3,607 & $-3,598$ & 0,000 & 0,27 \\
\hline Exploração Intencional-Sistemática (EIS) & 5,05 & 1,808 & 5,84 & 1,705 & $-5,713$ & 0,000 & 0,45 \\
\hline Quantidade de Informação (QI) & 9,30 & 1,944 & 9,76 & 2,011 & $-3,173$ & 0,002 & 0,23 \\
\hline Satisfação com a Informação (SI) & 9,32 & 1,988 & 9,78 & 1,940 & $-3,126$ & 0,002 & 0,23 \\
\hline Stresse com a Exploração (SE) & 15,23 & 4,325 & 15,80 & 4,318 & $-1,542$ & 0,125 & 0,13 \\
\hline Stresse com a Tomada de Decisão (STD) & 20,61 & 6,528 & 22,01 & 6,662 & $-2,639$ & 0,009 & 0,21 \\
\hline
\end{tabular}

IQE

Autonomia

Feedback dos colegas

$3,78 \quad 0,686$

Suporte social

$3,42 \quad 0,775$

Variedade de tarefas

$4,21 \quad 0,669$

Oportunidades de aprendizagem

$3,88 \quad 0,688$

Clareza Supervisor

$3,88 \quad 0,620$

Treino Supervisor

$3,63 \quad 0,760$

Feedback supervisor

$3,82 \quad 0,858$

$3,57 \quad 0,810$

Suporte do supervisor

$3,84 \quad 0,753$


Gamboa, V. M. \& Paixão, M. P. (2014). A Qualidade da Experiência de Estágio e o Desenvolvimento Vocacional de Estudantes dos Cursos Tecnológicos.

\section{Resultados}

A Tabela 1 mostra-nos que os valores médios das variáveis vocacionais sobem do primeiro (T1) para o segundo momento (T2), sendo exceção as subescalas Instrumentalidade externa (EE; $t=0,140 ; p=0,889 ; d=0,01)$, Instrumentalidade interna (II; $t=0,341 ; p=0,734 ; d=0,03$ ) e Importância da posição preferida (IPP; $t=2,487 ; p=0,014$; $d=0,19$ ), todas elas da dimensão crenças de exploração. Tomando apenas as mudanças cujas diferenças se revelaram estatisticamente significativas, podemos então afirmar que, entre o início e o fim do estágio (T1-T2), os alunos aumentaram os níveis de indecisão $(t=-3,012 ; p=0,003 ; d$ $=0,21)$ e reportaram crenças mais positivas relativamente aos resultados da exploração (CR $-t=-5,995 ; p=0,001$; $d=0,37)$ e ao estatuto do emprego $(t=0,213 ; p=0,831 ; d$ $=0,01)$, as quais foram acompanhadas de um incremento nos níveis de atividade exploratória $(\mathrm{EM}-t=-4,637 ; p$ $=0,001 ; d=0,33 ; \mathrm{ES}-t=-3,598 ; p=0,001 ; d=0,27$; EIS - $t=-5,713 ; p=0,001 ; d=0,45$; QI $-t=-3,173 ; p=$ 0,$002 ; d=0,23$ ) e nos níveis de satisfação (SI - $t=-3,126$; $p=0,002 ; d=0,23)$ e de stresse com a tomada de decisão $(t=-2,639 ; p=0,009 ; d=0,21)$. No sentido de explorar as diferenças nas variáveis psicológicas do domínio vocacional em função das variáveis sócio-demográficas, os resultados indicam que, no primeiro momento, as raparigas estão menos indecisas ( $\left.M_{\text {diferença }}=2,451, p=0,042\right)$, têm crenças de exploração mais favoráveis - Instrumentalidade externa (IE; $\left.M_{\text {diferença }}=-2,252, p=0,004\right)$ e Instrumentalidade interna (II; $\left.M_{\text {diferença }}=-1,380, p=0,001\right)$, e apresentam níveis mais elevados de exploração do meio (EM; $M_{\text {diferença }}=$ $-1,074, p=0,027)$. Depois do estágio, de um modo geral, os resultados continuam a ser favoráveis às raparigas, ainda que, ao nível da significância estatística, apenas se tenha observado diferenças na Instrumentalidade interna (II; $\left.M_{\text {diferença }}=-0,914, p=0,014\right)$. No que diz respeito à idade dos alunos, esta variável correlaciona positivamente com: as crenças de exploração vocacional (EE - T1 - $r=0,177, p$ $=0,013$; T2 $-r=0,168, p=0,018$; II - T2 - $r=0,184, p=$ $0,01)$, a exploração de si (ES; T2 - $r=0,174, p=0,014)$, a intencionalidade e sistematicidade da exploração (EIS; T2 - $r=0,263, p=0,001)$, o stresse com a tomada de decisão (T1 - $r=0,156, p=0,014$ ) e a tendência para a exclusão prematura de opções (TTF; T1 - $r=0,165, p=0,021)$. Já no que se refere ao nível socioeconómico (NSE) da família, importa destacar que são os alunos de NSE mais elevado que procuram informação de forma mais sistemática e intencional e também aqueles que apresentam crenças mais favoráveis relativamente aos resultados da exploração e ao estatuto do emprego.

A análise das relações entre todas as variáveis vocacionais estudadas (Tabela 2), revelou que entre momentos os valores encontrados sugerem, para o intervalo considerado, alguma estabilidade na atividade exploratória e nos níveis de indecisão e compromisso com as escolhas de carreira, uma vez que as correlações são moderadas e altamente significativas: (CDS) Indecisão - 0,51, (CCCS) Tendência para a Exclusão - 0,35, (CCCS) Exploração e Compromisso - 0,51, (CES) Estatuto do Emprego - 0,42, (CES) Certeza nos Resultados da Exploração - 0,44, (CES) Instrumentalidade Externa - 0,50, (CES) Instrumentalidade Interna-0,42, (CES) Importância Posição Preferida-0,45, (CES) Exploração do Meio - 0,41, (CES) Exploração de Si Próprio - 0,44, (CES) Exploração Sistemática e Intencional - 0,40, (CES) Quantidade de Informação - 0,49, (CES) Satisfação com a Informação - 0,45, (CES) Stresse na Exploração - 0,31, (CES) Stresse na Decisão - 0,37. Prosseguindo com a análise da Tabela 2, e agora para os valores apresentados abaixo (T1) e acima da diagonal (T2), constatamos que, globalmente, as diferentes dimensões vocacionais apresentam correlações moderadas e no sentido teoricamente esperado.

O próximo procedimento de análise de dados procura oferecer uma noção do impacto global do estágio nas dimensões vocacionais em estudo. Para tal, calcularam-se equações de regressão (hierárquica), nas quais as medidas vocacionais em T2 entraram na qualidade de variáveis dependentes, enquanto as obtidas em $\mathrm{T} 1$ foram incluídas no primeiro bloco, na qualidade de nível base do construto, de modo a se poder controlar o seu efeito. Num segundo passo, juntaram-se as variáveis sócio-demográficas (bloco II) e, num terceiro momento, entrou o bloco III, que integrou todas as variáveis relativas à qualidade do estágio. Desta forma, foi possível verificar se, no intervalo T1-T2, a qualidade do estágio teve algum impacto nas mudanças que se observaram nas variáveis vocacionais. Previamente, o estudo da intensidade da multicolinearidade das variáveis independentes, através da análise das correlações entre as variáveis em estudo, das estatísticas de colinearidade (Tolerância e VIF) e diagnóstico da colinearidade através do Condition Index e da proporção da variância, revelou que a intensidade da multicolinearidade é baixa, o que torna viável o cálculo das equações da regressão hierárquica. Pela leitura do resumo das análises que se apresenta na Tabela 3, podemos constatar, através dos valores das estatísticas da mudança, que a qualidade do estágio tem impacto na quase totalidade das variáveis do domínio vocacional, designadamente no Estatuto do emprego (EE; $\Delta R 2=5,7)$ e na Instrumentalidade externa (IE; $\Delta R 2=$ $8,9)$, da dimensão crenças de exploração, e nas variáveis Exploração do meio (EM; $\Delta R 2=8,7)$ e Exploração intencional e sistemática (EIS; $\Delta R 2=12,8$ ), da dimensão comportamentos de exploração vocacional. Verifica-se, igualmente, a partir dos valores do coeficiente de determinação da mudança, que a qualidade do estágio tem impacto na mudança ocorrida nos níveis de Stresse com a tomada de decisão $(\Delta R 2=5,2)$. No terceiro bloco, correspondente às medidas relativas à qualidade do estágio, a análise dos coeficientes de regressão para cada uma das variáveis, permite-nos identificar que, no que se refere aos níveis de indecisão (CDS), o valor preditivo recai nas variáveis Suporte social $(\beta=-0,143 ; t=-2,078 ; p=0,038)$ e Treino proporcionado pelo supervisor $(\beta=-0,210 ; t=-2,527$; $p=$ $0,012)$. No âmbito das crenças de exploração, os preditores 


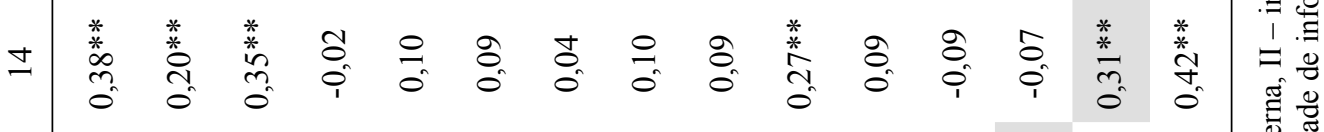

$=$ ר

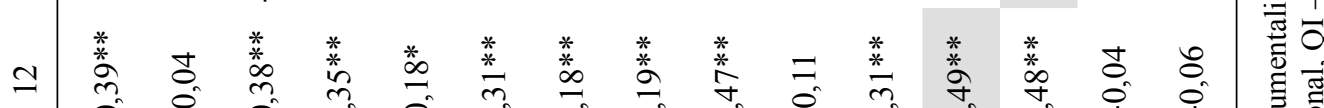

구에

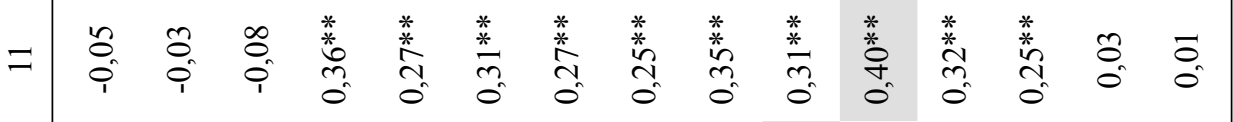

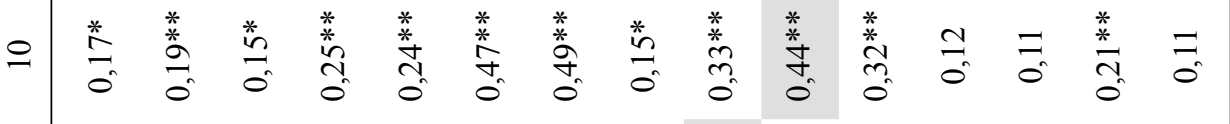

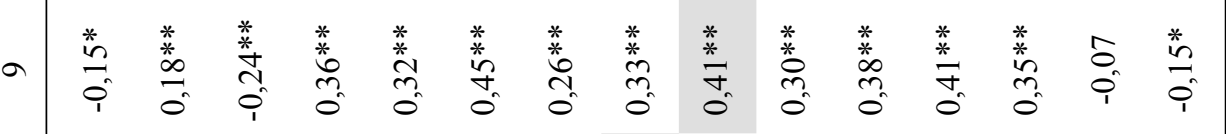

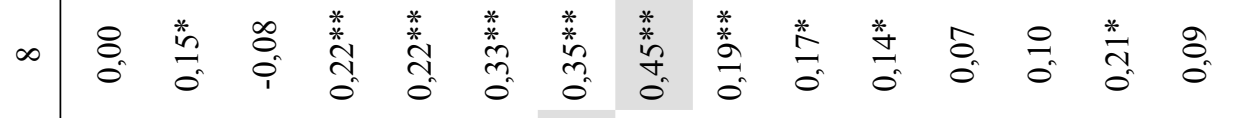

N

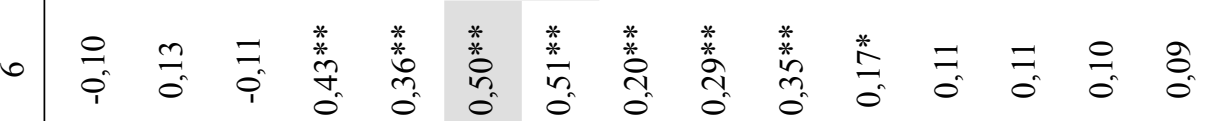

n

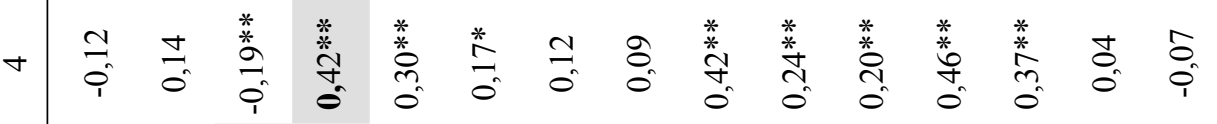

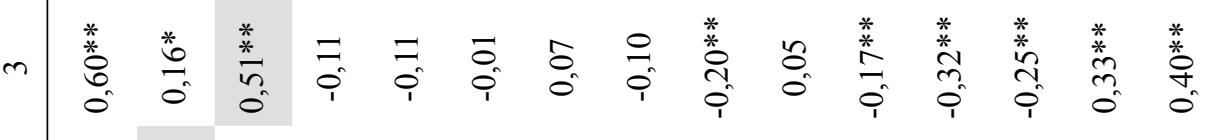

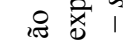

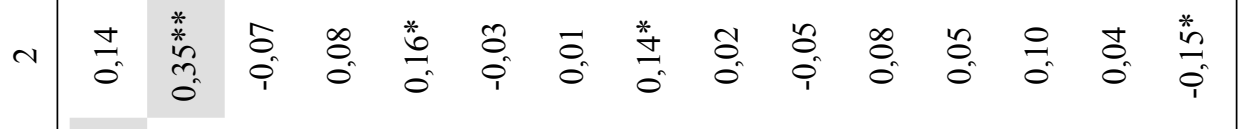

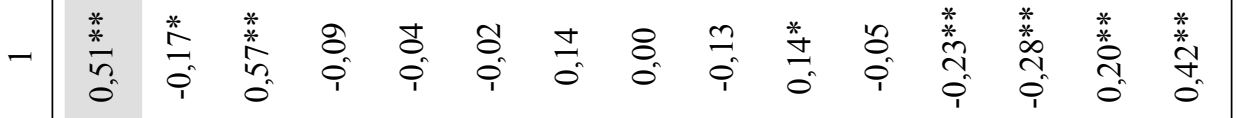


Gamboa, V. M. \& Paixão, M. P. (2014). A Qualidade da Experiência de Estágio e o Desenvolvimento Vocacional de Estudantes dos Cursos Tecnológicos.

Tabela 3

Síntese das Regressões Hierárquicas para Cálculo do Impacto da Qualidade do Estágio na Variação dos Valores Médios das Variáveis Vocacionais. (N=196)

\begin{tabular}{|c|c|c|c|c|c|}
\hline & \multirow{3}{*}{$\begin{array}{c}\text { Bloco I - } \\
\begin{array}{c}\text { Variável } \\
\text { em T1 }\end{array} \\
R 2\end{array}$} & \multirow{3}{*}{$\begin{array}{c}\text { Bloco II - Variável em T1 } \\
+ \text { sociodemográficas } \\
\text { (bloco de controlo) }\end{array}$} & \multirow{2}{*}{\multicolumn{3}{|c|}{$\begin{array}{c}\begin{array}{c}\text { Bloco III - Bloco I + Bloco II } \\
+ \text { Qualidades do estágio }\end{array} \\
\text { Estatísticas da mudança }\end{array}$}} \\
\hline & & & & & \\
\hline & & & $R 2$ & $\begin{array}{c}\Delta R 2 \\
\text { (Bloco II - Bloco III) }\end{array}$ & Sig. Ch. \\
\hline CDS- Indecisão & 25,2 & 25,6 & 30,0 & 4,3 & 0,019 \\
\hline CCCS- TTF & 13.2 & 13.4 & 18.0 & 4.6 & 0,032 \\
\hline CCCS-VEC & 20,2 & 21,5 & 26,4 & 4,9 & 0,010 \\
\hline Estatuto emprego (EE) & 13,6 & 15,8 & 21,5 & 5,7 & 0,006 \\
\hline Certeza resultados (CR) & 11,8 & 13,4 & 16,5 & 3,1 & ns \\
\hline Instrumentalidade externa (IE) & 22,2 & 24,6 & 33,5 & 8,9 & 0,000 \\
\hline Instrumentalidade interna (II) & 17,7 & 20,8 & 25,7 & 4,9 & 0,012 \\
\hline Importância posição (IPP) & 15,4 & 15,7 & 20,3 & 4,6 & 0,029 \\
\hline Exploração do meio (EM) & 19,5 & 21,7 & 30,4 & 8,7 & 0,000 \\
\hline Exploração de si (ES) & 19 & 21,5 & 25,6 & 4,1 & 0,044 \\
\hline Exploração I. Sistemática (EIS) & 11,9 & 17,4 & 30,2 & 12,8 & 0,000 \\
\hline Quantidade Informação (QI) & 24 & 25,8 & 29,4 & 3,5 & ns \\
\hline Satisfação informação (SI) & 14,6 & 15,3 & 19,6 & 4,3 & 0,042 \\
\hline Stresse exploração (SE) & 8,4 & 8,7 & 13,6 & 4,9 & 0,030 \\
\hline Stresse decisão (STD) & 13,8 & 14,3 & 19,5 & 5,2 & 0,013 \\
\hline
\end{tabular}

significativos são o Feedback do supervisor ( $\mathrm{a}=0,176 ; t$ $=2,043 ; p=0,042)$, no que se refere à certeza quanto aos resultados da exploração (CR), e o Feedback dos colegas $(\beta=0,131 ; t=2,233 ; p=0,026)$, quando nos reportamos ao estatuto do emprego (EE). Por outras palavras, parece que a informação proporcionada no contexto de estágio surge positivamente associada a crenças mais favoráveis no âmbito da exploração vocacional. Tomando os resultados encontrados nas variáveis relativas aos comportamentos de exploração, surgem como preditores significativos: o Feedback dos colegas, no que se refere à exploração do meio (EM; $\beta=0,129 ; t=2,376 ; p=0,018)$, à exploração de si (ES; $\beta=0,121 ; t=2,141 ; p=0,033)$ e à exploração intencional e sistemática (EIS; $\beta=0,107 ; t=1,965 ; p=$ $0,050)$, sendo que nesta última dimensão também surge o Feedback do supervisor $(\beta=0,191 ; t=2,418 ; p=0,016)$. Ainda no âmbito dos comportamentos de exploração, as oportunidades de aprendizagem predizem a exploração de si (ES; $\beta=0,168 ; t=2,214 ; p=0,028)$ e a autonomia prediz a exploração do meio (EM; $\beta=0,155 ; t=2,298 ; p$ $=0,022)$. Por último, no que se refere às reações de exploração, a clareza das instruções do supervisor surge como preditor significativo da variância observada no Stresse com a exploração $(\beta=0,249 ; t=2,762 ; p=0,006)$ e com a tomada de decisão $(\beta=0,202 ; t=2,320 ; p=0,021)$.

\section{Discussão dos Resultados}

A principal finalidade deste estudo foi analisar o impacto das qualidades do estágio curricular (experiência de trabalho) nos processos vocacionais de exploração, tomada de decisão e compromisso de carreira, em alunos dos cursos tecnológicos do ensino secundário português $\left(10^{\circ}, 11^{\circ}\right.$, $12^{\circ}$ anos de escolaridade). No que diz respeito à tomada de decisão e compromisso de carreira, das três medidas incluídas na avaliação destes construtos, apenas os valores médios da indecisão apresentam diferenças com significância estatística, no sentido de um aumento da indecisão 
no decurso do período de estágio (T1-T2). Tratando-se de cursos do ensino secundário, a conclusão dos mesmos e a realização do estágio têm lugar numa fase em que os alunos já estabeleceram um assinalável compromisso com as escolhas de carreira (e.g., Germeijs \& Verschueren, 2007a; Harren, 1979; Super et al., 1996), sobretudo devido ao cariz profissionalizante desta modalidade de formação. Neste sentido, e tendo em conta os níveis de compromisso reportados em ambos os momentos, podemos então admitir que, na maior parte dos alunos, a ação vocacional possa estar focalizada sobretudo nos processos de planeamento e implementação da escolha (e.g., Gati \& Asher, 2001; Germeijs \& Verschueren, 2007b; Porfeli \& Lee, 2012), e, não tanto, nos aspetos associados à seleção de uma alternativa de formação. No que concerne à exploração vocacional (pré e pós-teste), observaram-se ganhos com significado estatístico em nove das doze dimensões, sobretudo na atividade exploratória que se orientou para o meio (EM e ESI). Estes resultados vão ao encontro da expectativa teórica e de alguns estudos empíricos que suportam a ideia de que a transição para novos contextos pode produzir um incremento da atividade exploratória (e.g., Blustein, 1997a; Cheung \& Arnold, 2010; Flum \& Blustein, 2000; Jordaan, 1963; Kalakoski \& Nurmi, 1998; Savickas, 2005; Super et al., 1996; Taveira, 2001), eventualmente pela necessidade que os estagiários têm de dar sentido às experiências que estão a viver e garantir, dessa forma, um melhor ajustamento ao novo contexto de formação, a qual pode ser acompanhada de um aumento do stresse com a tomada de decisão, pois mesmos os processos de transição normativos e desejados, podem fazer-se acompanhar de um aumento da ansiedade relativa à exploração e à tomada de decisão (e.g., Blustein, 1997a; Jordaan, 1963; Kalakoski \& Nurmi, 1998; Super et al., 1996; Taveira, 1997, 2001). Ainda ao nível da significância estatística, mas em sentido contrário, observa-se uma descida dos valores médios da subescala importância da posição preferida (IPP; $M_{\text {diferença }}=$ $0,418, p=0,014)$. Efetivamente, por se tratar do primeiro contacto formal com a realidade ocupacional, podemos admitir que os constrangimentos inerentes ao mundo do trabalho (e.g., Watts, 1996) tenham um significativo impacto nos alunos dos cursos tecnológicos, uma vez que o ajustamento com o este novo contexto de aprendizagem não terá ocorrido de forma gradual (e.g., Dawis, 2005; Swanson \& Fouad, 1999).

Tomando agora as relações observadas entre as variáveis sócio-demográficas, são os participantes mais velhos (e.g., Rogers \& Creed, 2011; Rogers, Creed, \& Glendon, 2008), de nível socioeconómico mais elevado (e.g.; Blustein et al., 2002; Thompson \& Subich, 2006) e do sexo feminino (e.g., Creed et al., 2009; Kracke \& Schmitt-Rodermund, 2001; Rogers \& Creed, 2011) que reportam os maiores níveis de exploração, que se apresentam menos indecisos (e.g., Vondracek, Hostetler, Schulenberg, \& Shimizu, 1990), mais comprometidos (e.g., Chung, 2002) e com menor tendência para a exclusão prematura de opções (e.g., Lopez, 1994). Para o intervalo relativo ao período de estágio, as correlações entre os valores médios obtidos em cada um dos momentos (T1xT2) sugerem alguma estabilidade na atividade exploratória e nos níveis de indecisão, de compromisso e de autoeficácia. Na verdade, a literatura aponta o resultado apresentando em $\mathrm{T} 1$ como o melhor preditor de cada uma destas variáveis em T2 (e.g., Carless \& Prodan, 2003; Hirschi et al., 2011; Kracke \& Schmitt-Rodermund, 2001; Lent et al., 1994; Patton \& Creed, 2001; Rogers \& Creed, 2011) sobretudo quando nos aproximamos do fim da adolescência e assistimos a alguma estabilização dos processos vocacionais mais diretamente envolvidos na tomada de decisão e compromisso de carreira (Harren, 1979; Porfeli \& Lee, 2012; Skorikov, 2007; Super et al., 1996). No entanto, se globalmente a experiência de estágio tem impacto no desenvolvimento vocacional dos alunos, tal como é colocada, esta relação não responde à nossa principal questão - quais as qualidades do estágio que melhor explicam as mudanças ocorridas. Como resultados mais significativos, importa sublinhar que as qualidades supervisivas (treino, feedback e suporte), juntamente com o suporte social e o feedback dos colegas, surgem como as qualidades com maior impacto nas diversas dimensões da exploração vocacional, bem como nos níveis de indecisão e compromisso. No seu conjunto, estes resultados são consentâneos com os pressupostos das teorias relacionais do domínio vocacional, as quais salientam o papel facilitador do suporte social, da vinculação e do apoio emocional na exploração e na tomada de decisão (e.g., Blustein et al., 1995; Flum \& Blustein, 2000; Schultheiss, 2003); bem como com alguns estudos empíricos levados a cabo nos últimos anos (e.g., Creed et al., 2009; Hirschi, 2009; Hirschi et al., 2011; Kracke \& Schmitt-Rodermund, 2001; Ryan, Solberg, \& Brown, 1996), os quais têm vindo a conferir um suporte substancial às proposições avançadas na teoria. Assim, de acordo com a literatura, o suporte e os ensinamentos dos pares e supervisores podem representar efetivamente um fator facilitador na realização do estágio curricular e no próprio processo de desenvolvimento vocacional dos alunos, sobretudo se tivermos em consideração as descontinuidades e os desafios inerentes a uma situação de aprendizagem experiencial (e.g., Blustein, 1997b; Jordaan, 1963; Kuijpers \& Meijers, 2012; Petherbridge, 1996; Watts, 1996), sendo, neste caso, particularmente importantes os ensinamentos e o apoio dos professores/ supervisores (e.g., Cheung \& Arnold, 2010; Creed et al., 2009; Kenny \& Bledsoe, 2005; Lent, Hackett, \& Brown, 1999). Interessa ainda referir que, tal como se postula na teoria da autodeterminação (e.g., Ryan \& Deci, 2000), as competências desenvolvidas no contexto de estágio e a autonomia experimentada parecem ser relevantes na exploração do meio (EM e QI) e na exploração dirigida ao self (ES). 
Gamboa, V. M. \& Paixão, M. P. (2014). A Qualidade da Experiência de Estágio e o Desenvolvimento Vocacional de Estudantes dos Cursos Tecnológicos.

\section{Considerações Finais}

Uma primeira ideia a sublinhar relativamente aos principais resultados deste estudo, é que nos parece inquestionável a relevância da experiência de estágio no desenvolvimento vocacional dos alunos dos cursos tecnológicos do ensino secundário, sobretudo no âmbito dos comportamentos de exploração. Acresce, ainda, uma importante consideração acerca do papel desempenhado pelas diferentes qualidades do estágio. De facto, os resultados encontrados sugerem, na linha do pensamento de Tesluk e Jacobs (1998), que o impacto da experiência de aprendizagem em contexto real de trabalho depende das diferentes qualidades do contexto e das múltiplas possibilidades de interação que o mesmo oferece. Estes resultados vêm, do nosso ponto de vista, reforçar a natureza ecológica do desenvolvimento vocacional, pois apontam para a necessidade de, a exemplo do que sugere a perspetiva desenvolvimentista - contextualista (e.g., Vondracek et al., 1986; Vondracek \& Porfeli, 2008), se colocar o centro da análise no sistema de relações que se estabelecem entre o sujeito e o seu espaço relacional significativo. Em termos de implicações para a intervenção vocacional, o nosso principal contributo situa-se na rutura com a ideia de que o estágio, enquanto modalidade experiencial de aprendizagem, tem um impacto uniforme e sempre positivo na globalidade dos alunos. Por conseguinte, reafirmamos a importância das práticas diferenciadas no âmbito da intervenção vocacional. Por último, importa referir que o desenho de investigação adotado, com apenas dois momentos de recolha de dados, constitui uma forte limitação ao estudo, uma vez que apenas admitiu análises lineares das trajetórias que se desenharam entre o início e o fim do estágio.

\section{Referências}

Aryee, S., \& Tan, K. (1992). Antecedents and outcomes of career commitment. Journal of Vocational Behavior, 40, 288-305.

Blau, G. J. (1985). The measurement and prediction of career commitment. Journal of Occupational Psychology, 58, 277 288.

Blustein, D. L. (1997a). A context-rich perspective of career exploration across the life roles. The Career Development Quarterly, 45(3), 260-274.

Blustein, D. L. (1997b). The role of work in adolescent development. The Career Development Quarterly, 45(3), 381-389.

Blustein, D. L., Chaves, A. P. Diemer, M. A., Gallagher, L. A., Marshall, K. G., Sirin, S., \& Bhati, K. S. (2002). Voices of the 'Forgotten Half': The role of social class in the school-to-work transition. Journal of Counseling Psychology, 49, 311-323. doi:10.1037/0022-0167.49.3.311

Blustein, D. L., Ellis, M. V., \& Devenis, L. E. (1989). The development and validation of a two-dimensional model of the commitment to career choice process. Journal of Vocational Behavior, 35, 342-378.
Blustein, D. L., \& Flum, H. (1999). A self-determination perspective of interests and exploration in career development. In M. Savickas \& A. Spokane (Eds.), Vocational interests: Meaning, measurement, and counseling use (pp. 345-368). Palo Alto, CA: Consulting Psychologists Press.

Blustein, D. L., Prezioso, M., \& Schultheiss, D. (1995). Attachment theory and career development: Current status and future directions. The Counseling Psychologist, 23(3), 416-432.

Borges, N. (2007). Behavioral exploration of career and specialty choice in medical students. The Career Development Quarterly, 55(4), 351-358.

Bright, J., Pryor, R., Wilkenfeld, S., \& Earl, J. (2005). The role of social context and serendipitous events in career decision making. International Journal for Educational and Vocational Guidance, 5, 19-36. doi:10.1007/s10775-005-2123-6

Brooks, L., Cornelius, A., Greefield, E., \& Joseph, R. (1995). The relation of career-related work or internship experiences to the career development of college seniors. Journal of Vocational Behavior, 46, 332-349.

Carless, S. A., \& Prodan, O. (2003). The impact of practicum training on career and job search attitudes of postgraduate psychology students. Australian Journal of Psychology, 55, 89-94.

Cheung, R., \& Arnold, J. (2010). Antecedents of career exploration among Hong Kong Chinese university students: Testing contextual and developmental variables. Journal of Vocational Behavior, 76, 25-36. doi:org/10.1016/j.jvb.2009.05.006

Chung, Y. B. (2002). Career decision-making self-efficacy and career commitment: Gender and ethnic differences among college students. Journal of Career Development, 28(4), 277-284.

Creed, P., \& Patton, W. (2003). Differences in career attitude and career knowledge for high school students with and without paid work experience. International Journal for Educational and Vocational Guidance, 3, 21-33.

Creed, P., Fallon, T., \& Hood, M. (2009). The relationship between career adaptability, person and situation variables, and career concerns in young adults. Journal of Vocational Behavior, 74, 219-229. doi:org/10.1016/j.jvb.2008.12.004

Csikszentmihaly, M., \& Schneider, B. (2000). Becoming adult - How teenagers prepare for the world of work. New York: Basic Books.

Dawis, R. V. (2005). The Minnesota theory of work adjustment. In S. Brown \& R. Lent (Eds.), Career development and counseling: Putting research and theory to work (pp. 3-23). Hoboken, NJ: John Wiley \& Sons.

Flum, H., \& Blustein, D. (2000). Reinvigorating the study of vocational exploration: Framework for research. Journal of Vocational Behavior, 56, 380-404.

Gati, I., \& Asher, I. (2001). The PIC model for career decision making: Prescreening, in-depth exploration, and choice. In F. T. L. Leong \& A. Barak (Eds.), Contemporary models in vocational psychology: A volume in honor of Samuel $H$. Osipow (pp. 7-54). Hoboken, NJ: Lawrence Erlbaum.

Germeijs, V., \& Verschueren, K. (2007a). High school student's career decision-making process: Consequences for choice implementation in higher education. Journal of Vocational Behavior, 70, 223-241. doi:org/10.1016/j.jvb.2005.08.004

Germeijs, V., \& Verschueren, K. (2007b). Educational choices in adolescence: The decision-making process, antecedents and consequences. In V. Skorikov \& W. Patton (Eds.), Career development in childhood and adolescence (pp. 169-180). Rotterdam, Netherlands: Sense. 
Griffiths, T., \& Guile, D. (2004). Learning through work experience for the knowledge economy. Luxembourg City, Luxembourg: European Center for the Development of Vocational Training.

Halpern, R. (2012). Supporting vocationally oriented learning in the high school years: Rationale, tasks, challenges. New Directions for Youth Development, 134, 85-106. doi:10.1002/ yd.20018

Harren, V. A. (1979). A model of career decision making for college students. Journal of Vocational Behavior, 14, 119-133.

Hirschi, A. (2009). Career adaptability development in adolescence: Multiple predictors and effect on sense of power and life satisfaction. Journal of Vocational Behavior, 74, 145-155. doi:org/10.1016/j.jvb.2009.01.002

Hirschi, A., Niles, S., \& Akos, P. (2011). Engagement in adolescent career preparation: Social support, personality and the development of choice decidedness and congruence. Journal of Adolescence, 34, 173-182. doi:10.1016/j.adolescence.2009.12.009

Johnson, M. K., \& Mortimer, J. (2002). Career choice and development from a sociological perspective, In D. Brown (Ed.), Career choice and development (pp. 37-84). San Francisco. CA: Jossey-Bass.

Jordaan, J. P. (1963). Exploratory behavior: The formation of the self and occupational concepts. In D. Super, R. Starisshevsky, R. Matlin, \& J. P. Jordaan (Eds.), Career development: Self-concept theory (pp. 42-78). New York: College Entrance Board.

Kalakoski, V., \& Nurmi, J.-E. (1998). Identity and educational transitions: Age differences in adolescent exploration and commitment related to education, occupation, and family. Journal of Research on Adolescence, 8(1), 29-47.

Kenny, M. E., \& Bledsoe, M. (2005). Contributions of the relational context to career adaptability among urban adolescents. Journal of Vocational Behavior, 66(2), 257-272.

Kracke, B., \& Schmitt-Rodermund, E. (2001). Adolescents' career exploration in the context of educational and occupational transitions. In J.-E. Nurmi (Ed.), Navigating through adolescence: European perspectives (pp. 141-165). New York: Routledge Falmer.

Kuijpers, M., \& Meijers, F. (2012). Learning for now or later? Career competencies among students in higher vocational education in the Netherlands. Studies in Higher Education, 37, 449-467. doi:10.1080/03075079.2010.523144

Kuijpers, M., Meijers, F., \& Gundy, C. (2011). The relationship between learning environment and career competencies of students in vocational education. Journal of Vocational Behavior, 78, 21-30. doi:org/10.1016/j.jvb.2010.05.005

Larson, R., Wilson, S., \& Mortimer, J. (2002). Conclusions: Adolescents' preparation for the future. In R. Larson, B. Brown, \& J. Mortimer (Eds.), Adolescents' preparation for the future: A report of the study group on adolescence in the 21st century (pp. 159-166). Ann Arbor, MI: Blackwell.

Lent, R., Brown, S. D., \& Hackett, G. (1994). Toward a unifying social cognitive theory of career and academic interest, choice, and performance. Journal of Vocational Behavior, 45, 79-122.

Lent, R., Hackett, G., \& Brown, S. (1999). A social cognitive view of school-to-work transition. The Career Development Quarterly, 47, 297-311.

Lopez, F. G. (1994). Further validation of the commitment to career choices scale: Extensions to a high-school sample. Journal of Career Assessment, 2(4), 398-407.
Mortimer, J. (2003). Working and growing up in America. Cambridge, MA: Harvard University Press.

Osipow, S., Carney, C. G., Winer, J., Yanico, B., \& Koschier, M. (1976). The Career Decision Scale ( $3^{\text {rd }}$ rev. ed.). Columbus, $\mathrm{OH}$ : Marathon Consulting and Press.

Osipow, S., \& Winer, J. (1996). Career assessment and the Career Decision Scale. Journal of Career Assessment, 4, 117-130.

Patton, W., \& Creed, P. A. (2001). Developmental issues in career maturity and career decision status. The Career Development Quarterly, 49(4), 336-351.

Petherbridge, J. (1996). Debriefing work experience: A reflection on reflection? British Journal of Guidance and Counselling, 24(2), 243-257.

Porfeli, E., \& Lee, B. (2012). Career development during childhood and adolescence. New Directions for Youth Development, 134, 11-22. doi:10.1002/yd.20011

Rogers, M., \& Creed, P. (2011). A longitudinal examination of adolescent career planning and exploration using a social cognitive career theory framework. Journal of Adolescence, 34, 163-172. doi:10.1016/j.adolescence.2009.12.010

Rogers, M., Creed, P., \& Glendon, I. (2008). The role of personality in adolescent career planning and exploration: A social cognitive perspective. Journal of Vocational Behavior, 73(1), 132-142. doi:org/10.1016/j.jvb.2008.02.002

Ryan, R. E., \& Deci, E. (2000). Self-determination theory and the facilitation of intrinsic motivation, social development, and well-being. American Psychologist, 55(1), 68-78.

Ryan, N. E., Solberg, V. S., \& Brown, S. D. (1996). Family dysfunction, parental attachment, and career search self-efficacy among community college students. Journal of Counseling Psychology, 43(1), 84-89.

Savickas, M. L. (2005). The theory and practice of career construction. In S. D. Brown \& R. W. Lent (Eds.), Career development and counseling: Putting theory and research to work (pp. 42-70). Hoboken, NJ: John Wiley \& Sons.

Schultheiss, D. (2003). A relational approach to counseling: Theoretical integration and practical application. Journal of Counseling and Development, 81(3), 301-310.

Silva, J. T. (1997). Dimensões da indecisão da carreira. Investigação com adolescentes (Tese de doutorado, Universidade de Coimbra, Coimbra, Portugal).

Skorikov, V. (2007). Continuity in adolescent career preparation and its effects on adjustment. Journal of Vocational Behavior, 70, 8-24. doi:org/10.1016/j.jvb.2006.04.007

Skorikov, V., \& Vondracek, F. W. (1997). Longitudinal relationships between par-time work and career development in adolescents. The Career Development Quarterly, 45, 221-235.

Stone, J. R., \& Mortimer, J. (1998). The effect of adolescent employment on vocational development: Public and educational policy implications. Journal of Vocational Behavior, 53, 184-214.

Stumpf, S., Colarelli, S., \& Hartman, K. (1983). Development of the career exploration survey (CES). Journal of Vocational Behavior, 22, 191-226.

Super, D., Savickas, M. L., \& Super, C. M. (1996). The life-span, life-space approach to careers. In D. Brown \& L. Brooks (Eds.), Career choice and development. San Francisco, CA: Jossey-Bass.

Swanson, J. L., \& Fouad, N. A. (1999). Applying theories of person-environment fit to the transition from school to work. The Career Development Quarterly, 47, 337-347. 
Gamboa, V. M. \& Paixão, M. P. (2014). A Qualidade da Experiência de Estágio e o Desenvolvimento Vocacional de Estudantes dos Cursos Tecnológicos.

Taveira, M. C. (1997). Exploração e desenvolvimento vocacional de jovens. Estudo sobre as relações entre a exploração, a identidade e a indecisão (Tese de doutorado, Universidade do Minho, Braga, Portugal).

Taveira, M. C. (2001). Exploração vocacional: Teoria, investigação e prática. Psychologica, 26, 55-77.

Tesluk, P. E., \& Jacobs, R. (1998). Toward an integrated model of work experience. Personnel Psychology, 51, 321-354.

Thompson, M., \& Subich, L. (2006). The relation of social status to the career decision-making process. Journal of Vocational Behavior, 69, 289-301.

Vianen, A. E. M. V., Pater, I. E. D., \& Preenen, P. T. Y. (2009). Adaptable careers: Maximizing less and exploring more. The Career Development Quarterly, 57(4), 298-309.

Vondracek, F. W., Hostetler, M., Schulenberg, J., \& Shimizu, K. (1990). Dimensions of career indecision. Journal of Counseling Psychology, 37(1), 98-106.

Vondracek, F. W., Lerner, R., \& Schulenberg, J. (1986). Career development: A life-span developmental approach. London: Lawrence Erlbaum.

Vondracek, F. W., \& Porfeli, E. J. (2008). Social contexts for career guidance throughout the world. Developmental-contextual perspectives on career across the lifespan. In J. A. Athanasou \& R. V. Esboreck (Eds.), International handbook of career guidance (pp. 209-225): London: Springer Science and Business Media.

Watts, A. G. (1996). Experienced-based learning about work. In A. G. Watts, B. Law, J. Killeen, J. Kidd, \& R. Hawthorn (Eds.), Rethinking careers education and guidance: Theory, policy and practice (pp. 233-246). London: Routledge. 